\title{
Is It Possible to Reduce Even More Need for Axillary Dissection?
}

\author{
Georgios Pechlivanides, Depy Vassilarou, Stamatis Vassilaros \\ Prolipsis Breast Unit, Athens, Greece \\ Email: geopech@otenet.gr
}

Received December 27, 2012; revised January 30, 2013; accepted February 8, 2013

Copyright (C) 2013 Georgios Pechlivanides et al. This is an open access article distributed under the Creative Commons Attribution License, which permits unrestricted use, distribution, and reproduction in any medium, provided the original work is properly cited.

\begin{abstract}
It has always been disappointing to receive a histology report after an axillary dissection (ALND) saying that the only positive lymph node was the sentinel lymph node (SLN). It is for this reason that there have been many efforts to create the best predictive model in order to avoid non sentinel node dissection and in fact there are in use many of them with a reasonable success rate. The publication of the multicenter study by Giuliano et al. showed a disease free survival and overall survival rate equal between patients with positive SLN with or without axillary dissection in a large group of patients. Breast surgeons around the world have long before been interested in reducing even more the need for axillary lymph node dissection, so they easily grasped the chance of those results and applied them in their practice. Objections have been expressed regarding the integrity of the study methods and the results which make the need for a second study to confirm those results absolutely necessary.
\end{abstract}

Keywords: Sentinel Lypmh Node; Axillary Dissection; Breast Cancer

\section{Introduction}

More than 20 years after the introduction of sentinel node biopsy which was applied in order to avoid axillary lymph node dissection (ALND) in every breast cancer patient, but the positive SN patients, even this last principle has been challenged. At first it was challenged by many predictive of the non sentinel nodes status models that have been used the last ten years, some of them also available for application online, and recently by the Z0011 study of ACSOG [1].

It is well shown that approximately $60 \%$ of the patients with a sentinel node positive T1 tumor have a negative axilla and only around $10 \%$ will have four or more positive nodes, which would alter patient's management. Sentinel node positive T2 tumor patients have a negative axilla in $50 \%$ of cases. It seems reasonable to think that a considerable number of sentinel node positive patients have nothing to gain from ALND and they are put at risk for its rather serious complications.

Gerber et al. summarized the pros and contras for axillary lymph node status and associated treatment decisions [2]. According to them reasons for ALND could be: the treatment mismatch if node status is unknown (especially in ER-positive, HER2-negative disease), the use of dose-dense and dose-induced chemotherapy in patients with 4 or more involved lymph nodes, the resection of metastases as a potential source of distant metastases, the importance of tumor volume reduction, that nodal status remains an indicator for or against radiotherapy after mastectomy, and that it is necessary for defining $\mathrm{pN}$, for staging, study inclusion, and comparison of outcome. Reasons against ALND could be: that tumor biology is more important than nodal status regarding prognosis and treatment, that any axillary surgery increases costs and morbidity, that axillary recurrences are very seldom even in node positive patients, that delayed surgery of involved lymph nodes is without overall survival disadvantages, that distant metastasis may develop even in pN0 patients, that seldom exist axillary metastases in patients with medial tumor location, that systemic treatment acts also in axillary lymph node metastases, that there is no evidence that lymph node metastases are able to metastasize and that leaving involved lymph nodes in situ does not increase axillary recurrences or metastasis.

\section{Minimum Local Control Benefit from}

\section{ALND}

Two randomized trials investigated the use of ALND versus no axillary surgery in elderly patients $[3,4]$. A third trial randomized patients without any axillary sur- 
gery to no axillary treatment versus axillary radiotherapy [5]. The results of all three studies showed a very low rate of axillary recurrences, even in the arms without axillary surgery, and comparable disease-free and overall survival. In an observational study with 671 consecutive patients, aged $\geq 70$ years, and a clinically tumor-free axilla, 172 received and 499 did not receive axillary dissection [6]. After a median follow-up of 15 years, there was no significant difference in breast cancer mortality between the axillary and no axillary clearance groups. Ipsilateral axillary recurrence rate was zero in the axillary dissection group and relatively low in the no axillary dissection group: $5.8 \%$ overall and $3.7 \%$ for pT1 patients.

From the study Z0011 of ACSOG, when they first looked for locoregional recurrence at 6.3 years of follow up, found no significant difference in local ( $p$ 0.11) or regional recurrence ( $\mathrm{p}$ 0.45) [7]. All evidence existing to now point towards little or no benefit of ALND for local control.

\section{Involved Axillary Nodes as Possible Source of Metastases}

This question has not been answered yet but there is increasing doubt, that lymph node metastasis are able to metastasize. Among several theories of infiltrated lymph nodes being a step towards remote metastases, it has been proved that high levels of VEGF-C and VEGFR-3 are not only associated with an increase of lymph node metastasis but also with poor overall survival without affecting the growth of the primary tumor. VEGF-Cinduced lymphangiogenesis in SLN promotes tumor metastasis spread to distant sites $[8,9]$. These results implicate the possibility of further metastasis to distant organs from the lymph nodes via the thoracic duct. One of the most strong objections against is that a significant number of patients show discordant quantitative expression of molecular markers between primary and nodal disease indicating an organotropy [10]. This concept of organ site specificity corroborates the hypothesis that lymph node metastases do not further spread or if they do only into lymph nodes down stream the metastatic one. In daily practice now it has been shown in several studies, as it was mentioned already, that there is no relation between omitting ALND and worse survival. It was clear also in the study of Martelli et al. that the incidence of metastases was slightly higher, but not statistically significant, in elderly patients treated with ALND (11.6\% vs 9.9\%) [6]. The study of Avril et al. in which 625 women aged $\geq 50$ years with early breast cancer (tumor $\leq 10 \mathrm{~mm}$ ) and clinically-negative axillary nodes were randomized to receive treatment with $\operatorname{ALND}(\mathrm{Ax})$ or without (no-Ax) failed to reproduce the above results [11]. OS and DFS at five years were not equivalent (Ax vs. no-Ax: 98\% vs.
$94 \%$ and $96 \%$ vs $90 \%$ respectively). Recurrence was higher for no-Ax, particularly in the first five years after surgery. Axillary nodes were positive for $14 \%$ Ax patients but only $2 \%$ no-Ax patients experienced axillary node recurrence. Several interpretations were proposed by the authors for the slight advantage in OS at five years for ALND patients. They suggested either a positive effect of the chemotherapy received by $8 \%$ Ax patients (vs. $2 \%$ of no-Ax patients), or adverse effects of the endocrine therapy received by the majority of the no-Ax group. Involved axillary nodes are still questioned as possible source of metastases.

\section{Information Provided by ALND}

The only information gained from ALND is the number of positive lymph nodes but with a considerable increase of morbidity. Relying on clinical examination for diagnosis of an involved node is not safe as its sensitivity to detect disease is reported at 25\% - 35.5\% [12-14].

Buruah et al. found that preoperative ultrasound with the addition of FNA when there is suspicion for metastatic infiltration might spare $28.5 \%$ of patients from the need for SLNB [15]. The sensitivity of axillary ultrasound combined with FNA is low $(53 \%-59 \%)$ but the specificity is $100 \%[16,17]$. Possibly because the criteria for node infiltration are still not clear. More reliable criteria seem to be the cortex thickening $(>2.5 \mathrm{~mm})$ and the absence of fatty hilum [18]. There are promising data showing that contrast enhanced and power doppler ultrasound can differentiate more accurately between benign and malignant lymph nodes in breast cancer [19]. Malignant lymph nodes demonstrated longer contrast enhancement duration compared to benign lymph nodes.

The diagnostic accuracy of FDG-PET/CT for the detection of axillary lymph node metastases in breast cancer patients acquired 60 min after FDG administration is reported to be only moderate, especially due to low sensitivity. Relevant studies examined identified that FDG$\mathrm{PET} / \mathrm{CT}$ has a sensitivity of $60 \%$ and a specificity of $97 \%$ in detecting lymphatic metastasis. Although positive axillary FDG-PET/CT is a good predictor of axillary disease and correlates well with SLNB, the relatively poor sensitivity $(60 \%)$ must be considered for treatment planning [20]. Metabolic activity varies according to the histopathology type.

A recent review evaluated the diagnostic accuracy of magnetic resonance imaging (MRI) techniques for identification of axillary metastases in early stage newly diagnosed breast cancer patients [21]. Based on the highest sensitivity and specificity reported in each of the nine studies evaluating ultra small super-paramagnetic iron oxide (USPIO)-enhanced, gadolinium-enhanced MRI and in-vivo proton MR spectroscopy mean sensitivity was $90 \%$ and mean specificity $90 \%$. The best results 
were produced by USPIO enhanced MRI, with a mean sensitivity $98 \%$ and a mean specificity $96 \%$. Its accuracy is related to the histopathology type and isn't able to reveal micrometastases.

The combination of all available modalities and physical examination cannot offer a sensitivity of more than $56.7 \%$ as it was shown in the study of Valente et al. [12]. So far we cannot obtain by other means the information provided by ALND. It is pragmatic though, to believe that in the near future the technology will offer an acceptable and safe for the patient tool for the accurate estimation of lymph node status.

\section{Patient Management Based on Number of Positive Nodes}

Lymph node status has been the basis for prognosis and treatment decision in breast cancer for many years. In the most recent years it is believed that lymph node status is a good indicator of tumor stage related to the time point of diagnosis, but it reflects neither the real tumor biology and metastatic behaviour nor the sensitivity to systemic treatment, according to Gerber et al. [2]. Axillary lymph node involvement is the result of different factors and lymph node status at all does not reflect the aggressive behaviour of the tumor correctly [22]. Poor outcome depends more on the tumor biology than on lymph node involvement [23,24]. Gene expression analyses of the primary tumor indicated that $51 \%$ of lymph node-negative tumors and $49 \%$ of lymph node-positive tumors have a poor prognostic signature [25].

Guidelines from 2011 St. Gallen Breast Cancer Conference consensus don't suggest a change of adjuvant systemic treatment based on the number of positive lymph nodes, except from very unusual sub-groups of breast cancer [26]. The consensus panel did not believe that node positivity per se is an indication for use of chemotherapy, though a strong majority would use it if more than three lymph nodes were involved. They also voted in favor of post mastectomy radiotherapy if more than 3 nodes are affected. IBCSG published in 2012 the evaluation of 8106 patients enrolled in 13 randomized trials and concluded that radiotherapy should be delivered to the chest wall and supraclavicular fossa post mastectomy if 4 or more axillary nodes are infiltrated or in case of $1-3$ involved nodes when the patient is young $(<40 \mathrm{y})$, if total number of lymph nodes is less than 7 or if there is vascular invasion [27].

Thus the number of infiltrated nodes has still a significant role for the radiotherapy treatment decision and a role for systemic treatment in small groups of patients.

\section{Axillary Clearance Doesn't Change the Outcome}

From the previously presented studies of Martelli et al.,
Rudendam et al. and $\mathrm{V}$ or radiotherapy versu eronesi et al. which compared axillary dissection or nothing regardless of sentinel node biopsy, there was no significant difference in overall survival and disease free survival [3-5]. Similarly a previous study (NSABBP04) which compared total mastectomy and radiotherapy of the axilla versus radiotherapy of the axilla in case of recurrence, found no outcome difference after a mean 25 year follow up [28]. In the years followed those studies, sentinel lymph node biopsy enabled us to select the patients with positive frozen section for axillary dissection. The aim of multicentric Z0011 study of ACSOG was to move beyond SLNB and disengage the positivity of SLN from the axillary dissection [1]. The study came to an early close due to reduced mortality and published in 2011 the results of 891 patients with T1-2, cN0 cancer with infiltration of 1 or 2 sentinel nodes (imprints or H\&E) subjected to lumpectomy and radiotherapy. Patients were randomized to either ALND or not and they were followed for a mean 6.3 years. Five year total survival rate was $91.8 \%$ for the group of ALND vs $92.5 \%$ for the SLNB-only group. Five year disease free survival was $82.2 \%$ vs $83.9 \%$ respectively (Figure 1). The hazard ratio for treatment-related overall survival was 0.79 without adjustment and 0.87 after adjusting for age and adjuvant therapy. However, several objections have been expressed for the technical integrity of this study, the most comprehensive report being the one by the CAGS/ ACS Evidence Based Reviews in Surgery Group from Canada [29]. The margin of noninferiority in the trial by Giuliano and colleagues was based on the SLND group having a 5-year survival of "not less than $75 \%$ of that observed in the ALND group." The expected 5-year survival of the ALND group was estimated to be $80 \%$. Thus, based on this statement, an absolute 5-year survival as low as $60 \%$ in the SLND group, a $20 \%$ absolute difference from the ALND group would be sufficient to conclude that SLND is not inferior to ALND. This margin of noninferiority would be unacceptable to most clinicians and patients. The trial was closed early with less than $50 \%$ of the targeted accrual as accrual was slow and there was a substantially higher than expected survival in the pooled data from the 2 groups. It was estimated that even with all 1900 targeted patients accrued, it would take 20 years of follow-up to observe the estimated 500 deaths needed to declare non-inferiority. Instead the results of the trial were reported with only 92 events. There were many patients entered into the trial who had no positive nodes; 29 (7.0\%) patients in the SLND group. There were also a large number of women lost to follow up: $21 \%$ in the ALND group and $17 \%$ in the SLND group and it is well known that when the number lost to follow-up exceeds $10 \%$, the validity of the conclusions may be jeopardized. There were a large number of pa- 

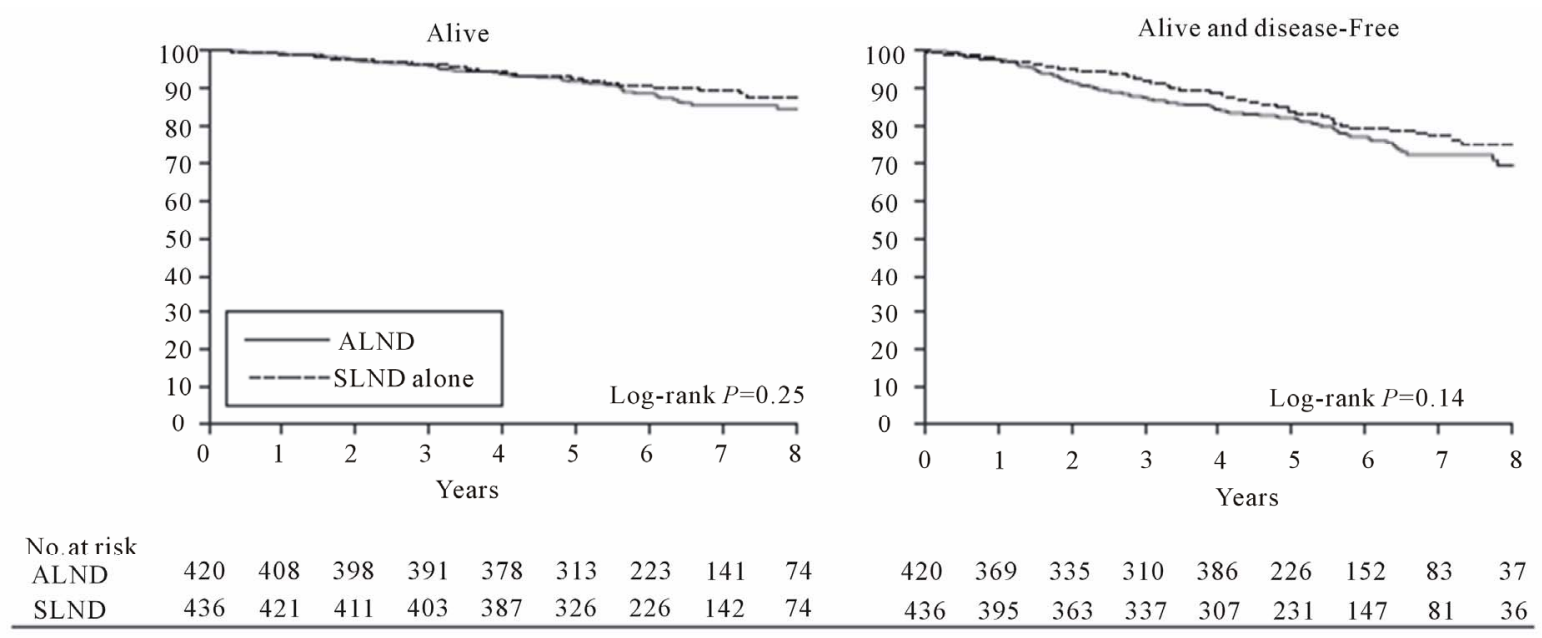

\begin{tabular}{|c|c|c|c|c|c|c|c|c|c|c|c|c|c|c|c|c|c|c|}
\hline $\begin{array}{l}\text { No. at risk } \\
\text { ALND }\end{array}$ & 420 & 408 & 398 & 391 & 378 & 313 & 223 & 141 & 74 & 420 & 369 & 335 & 310 & 386 & 226 & 152 & 83 & 37 \\
\hline SLND & 436 & 421 & 411 & 403 & 387 & 326 & 226 & 142 & 74 & 436 & 395 & 363 & 337 & 307 & 231 & 147 & 81 & 36 \\
\hline
\end{tabular}

ALND indicates axillary lymph node dissection; SLND, sentinel lymph node dissection.

Figure 1. Survival of the ALND group compared with SLND-alone group [1].

tients for whom there are no data on the number of positive nodes: $18 \%$ in the ALND group. The dosing, frequency and field definition guidelines of radiation treatment were not described and there were 43 (5.0\%) patients who did not receive their assigned treatment. Furthermore, patients in the SLN biopsy-only arm had slightly better prognostic factors overall, with a higher proportion of micrometastases in the SLN biopsy arm (45\%) compared with the ALND arm (35\%).

The recently presented MA-20 trial, in contrast to Z0011 showed that, by adding to the standard WBI, irradiation to internal mammary, supraclavicular and high axillary nodes for patients with positive nodes and segmental mastectomy results to significant improvement of DFS and a small improvement of overall survival [30]. After its preliminary results, relevant results are pending from the AMAROS study which randomized patients with sentinel nodes to ALND or axillary radiotherapy [31].

Many surgeons have changed their practice to a more selective use of ALND during recent years and for sure long before the Z0011 study results been presented. MSKCC reviewed their prospective SLN database over 10 years (1997-2006, 7509 SLN procedures) for time trends and variation between surgeons in the use of SLN-FS and ALND in patients with cNO invasive breast cancer [32]. They found that the use of SLN-FS decreased from $100 \%$ to $62 \%$ and varied widely by surgeon (66\% to $95 \%)$. There was statistically significant trend in the performance of ALND for patients with SLN metastases only for those detected by serial section H\&E with or without immunohistochemistry. These trends coincided with an increase in the proportion of completion versus immediate ALND. Those findings suggest a more nuanced approach to axillary management. They also calculated that if the Z0011 selection criteria had been applied to their cohort, $66 \%$ of SLN-FS and $48 \%$ of ALND would have been avoided, sparing $13 \%$ of all patients the morbidity of ALND.

Axillary clearance, when a sentinel node is positive does not change the outcome of almost all patients. It seems that a substantial number of patients will have mostly their disease free survival been improved by adding an axillary clearance to a positive sentinel node biopsy. We need to accurately select this group of patients.

\section{Criteria Needed}

Predictive models for the possibility a non sentinel axillary lymph node being infiltrated, when the sentinel node is positive, have been used for some years now. Those predictive models used different combinations of clinical, radiological, histological and more recently molecular information for the tumour and the patient. Some of them are available in the internet with most reliable results being those of MSKCC, Stanford and Cambridge models. A meta-analysis showed that there is an unacceptably high variability in individual predictions when the nine predictive systems that were available were used to predict non-SLN metastasis in patients with SLN-positive breast cancer. No patients were uniformly classified as low risk by all nine prediction models [33]. At the group level, a considerable variation in the distribution of the predicted probabilities was observed. At the individual level, calculation of the predicted probabilities for the selected patients who were considered low risk $(\leq 10 \%)$ according to the MSKCC nomogram, showed even larger variations, ranging from $4 \%$ to $94 \%$.

More reliable have been proved the Z0011 trial of the American College of Surgeons Oncology Group (ACOSOG) 
criteria used to select patients who will not have an ALND: clinical T1-T2 invasive breast cancer, no palpable adenopathy, and 1 to 2 SLNs containing metastases identified by frozen section, touch preparation, or hematoxylin-eosin staining on permanent section treated with breast conservation, whole breast radiotherapy and adjuvant chemotherapy. The selected patients had no difference long term in both local and distant recurrence rate. Seven tested prediction models were not able to accurately identify low risk women from a cohort that would have been excluded from Z0011 [34].

Z0011 trial has agitated breast surgeons but has not changed the rules. At the Second Kyoto Breast Cancer Consensus Conference (KBCCC-Kyoto, Japan, April 14$16,2011)$ the consensus opinion supported omission of ALND in breast-conservation therapy patients with T1/ T2 tumors and micrometastases only in the SLN and perhaps macrometastases when the metastatic ratio is low-say one out of two or two out of four nodes, rather than one out of one or two out of two nodes.

More important is what Giuliano AE [35] stated recently that: "Currently, there are no definable criteria that completely prevent patients from benefiting from ALND in the setting of metastatic disease to the axilla. It is, therefore, clear that ALND should be strongly considered in the management of the SLN-positive axilla. SLN resection has its role in the management of early stage breast cancer, but whether SLN resection alone should replace ALND is yet to be determined."

We should better define the criteria by which to select the patient with positive sentinel node for ALND. For sure Z0011 trial results need confirmation by at least another, well designed and executed, trial in order to be adopted by all breast surgeons.

\section{REFERENCES}

[1] A. E. Giuliano, K. K. Hunt, K. V. Ballman, P. D. Beitsch, P. W. Whitworth, P. W. Blumencranz, A. M. Leitch, S. Saha, L. M. McCall and M. Morrow, "Axillary Dissection vs No Axillary Dissection in Women with Invasive Breast Cancer Andsentinel Node Metastasis: A Randomized Clinical Trial," JAMA, Vol. 305, No. 6, 2011, pp. 569-575. doi:10.1001/jama.2011.90

[2] B. Gerber, K. Heintze, J. Stubert, M. Dieterich, S. Hartmann, A. Stachs and T. Reimer, "Axillary Lymph Node Dissection in Early-Stage Invasive Breast Cancer: Is It Still Standard Today?" Breast Cancer Research and Treatment, Vol. 128, No. 3, 2011, pp. 613-624. doi:10.1007/s10549-011-1532-0

[3] G. Martelli, P. Boracchi, M. De Palo, S. Pilotti, S. Oriana, R. Zucali, et al., "A Randomized Trial Comparing Axillary Dissection to No Axillary Dissectionin Older Patients with T1N0 Breast Cancer: Results after 5 Years of Follow-Up," Ann Surgery, Vol. 242, No. 1, 2005, pp. 1-6. doi:10.1097/01.sla.0000167759.15670.14
[4] International Breast Cancer Study Group, C. M. Rudenstam, D. Zahrieh, J. F. Forbes, D. Crivellari, S. B. Holmberg, P. Rey, D. Dent, I. Campbell, J. Bernhard, K. N. Price, M. Castiglione-Gertsch, A. Goldhirsch, R. D. Gelber and A. S. Coates, "Randomized Trial Comparing Axillary Clearance versus No Axillary Clearance in Older Patients with Breast Cancer: First Results of International Breast Cancer Study Group Trial 10-93," Journal of Clinical Oncology, Vol. 24, No. 3, 2006, pp. 337-344. doi:10.1200/JCO.2005.01.5784

[5] U. Veronesi, R. Orecchia, S. Zurrida, et al., "Avoiding Axillary Dissection in Breast Cancer Surgery: A Randomized Trial to Assess the Role of Axillary Radiotherapy," Annals of Oncology, Vol. 16, No. 3, 2005, pp. 383388. doi:10.1093/annonc/mdi089

[6] G. Martelli, R. Miceli, M. G. Daidone, G. Vetrella, A. M. Cerrotta, D. Piromalli and R. Agresti, "Axillary Dissection versus No Axillary Dissection in Elderly Patients with Breast Cancer and No Palpable Axillary Nodes: Results after 15 Years of Follow-Up," Annals of Surgical Oncology, Vol. 18, No. 1, 2011, pp. 25-33. doi:10.1245/s10434-010-1217-7

[7] A. E. Giuliano, L. McCall, P. Beitsch, P. W. Whitworth, P. Blumencranz, A. M. Leitch, S. Saha, K. K. Hunt, M. Morrow and K. Ballman, "Locoregional Recurrence after Sentinel Lymph Node Dissection with or without Axillary Dissection in Patients with Sentinel Lymph Node Metastases: The American College of Surgeons Oncology Group Z0011 Randomized Trial," Annals of Surgery, Vol. 252, No. 3, 2010, pp. 426-432.

[8] R. A. Mohammed, A. Green, S. El Shikh, et al., "Prognostic Significance of Vascular Endothelial Cell Growth Factors-A, -C and -D in Breast Cancer and Their Relationship with Angio- and Lymphangiogenesis," British Journal of Cancer, Vol. 96, No. 7, 2007, pp. 1092-1100. doi:10.1038/sj.bjc.6603678

[9] Y. Nakamura, H. Yasuoka, M. Tsujimoto, et al., "Lymph Vessel Density Correlates with Nodal Status, VEGF-C Expression, and Prognosis in Breast Cancer," Breast Cancer Research and Treatment, Vol. 91, No. 2, 2005, pp. 125-132. doi:10.1007/s10549-004-5783-X

[10] S. J. Aitken, J. S. Thomas, S. P. Langdon, et al., "Quantitative Analysis of Changes in ER, PR and HER2 Expression in Primary Breast Cancer and Paired Nodal Metastases," Annals of Oncology, Vol. 21, No. 6, 2010, pp. 1254 1261. doi:10.1093/annonc/mdp427

[11] A. Avril, G. Le Bouëdec, G. Lorimier, J. M. Classe, C. Tunonde-Lara, S. Giard, G. MacGrogan, M. Debled, S. Mathoulin-Pélissier and L. Mauriac, "Phase III Randomized Equivalence Trial of Early Breast Cancer Treatments with or without Axillary Clearance in Post-Menopausal Patients Results after 5 Years of Follow-Up," European Journal of Surgical Oncology, Vol. 37, No. 7, 2011, pp. 563-570. doi:10.1016/j.ejso.2011.04.008

[12] S. A. Valente, G. M. Levine, M. J. Silverstein, J. A. Rayhanabad, J. G. Weng-Grumley, L. Ji, D. R. Holmes, R. Sposto and S. F. Sener, "Accuracy of Predicting Axillary Lymph Node Positivity by Physical Examination, Mammography, Ultrasonography, and Magnetic Resonance Imaging," Annals of Surgical Oncology, Vol. 19, No. 6, 
2012, pp. 1825-1830. doi:10.1245/s10434-011-2200-7

[13] M. Pamilo, M. Soiva and E. M. Lavast, "Real-Time Ultrasound, Axillary Mammography, and Clinical Examination in the Detection of Axillary Lymph Node Metastases in Breast Cancer Patients," Journal of Ultrasound in Medicine, Vol. 8, No. 3, 1989, pp. 115-120.

[14] K. A. Kvistad, J. Rydland, H. B. Smethurst, S. Lundgren, H. E. Fjosne and O. Haraldseth, "Axillary Lymph Node Metastases in Breast Cancer: Preoperative Detection with Dynamic Contrast-Enhanced MRI," European Radiology, Vol. 10, No. 9, 2000, pp. 1464-1471. doi: $10.1007 / \mathrm{s} 003300000370$

[15] B. P. Baruah, A. Goyal, P. Young, A. G. Douglas-Jones and R. E. Mansel, "Axillary Node Staging by Ultrasonography and Fine-Needle Aspiration Cytology in Patients with Breast Cancer," British Journal of Surgery, Vol. 97, No. 5, 2010, pp. 680-683. doi:10.1002/bjs.6964

[16] A. García Fernández, M. Fraile, N. Giménez, A. Reñe, M. Torras, L. Canales, J. Torres, I. Barco, S. González, E. Veloso, C. González, L. Cirera and A. Pessarrodona, "Use of Axillary Ultrasound, Ultrasound-Fine Needle Aspiration Biopsy and Magnetic Resonance Imaging in the Preoperative Triage of Breast Cancer Patients Considered for Sentinel Node Biopsy," Ultrasound in Medicine \& Biology, Vol. 37, No. 1, 2011, pp. 16-22.

doi:10.1016/j.ultrasmedbio.2010.10.011

[17] M. B. Mainiero, C. M. Cinelli, S. L. Koelliker, T. A. Graves and M. A. Chung, "Axillary Ultrasound and FineNeedle Aspiration in the Preoperative Evaluation of the Breast Cancer Patient: An Algorithm Based on Tumor Size and Lymph Node Appearance," American Journal of Roentgenology, Vol. 195, No. 5, 2010, pp. 1261-1267. doi:10.2214/AJR.10.4414

[18] N. Cho, W. K. Moon, W. Han, I. A. Park, J. Cho and D. Y. Noh, "Preoperative Sonographic Classification of Axillary Lymph Nodes in Patients with Breast Cancer: Node-to-Node Correlation with Surgical Histology and Sentinel Node Biopsy Results," American Journal of Roentgenology, Vol. 193, No. 6, 2009, pp. 1731-1737. doi:10.2214/AJR.09.3122

[19] I. Steppan, D. Reimer, E. Müller-Holzner, C. Marth, F. Aigner, F. Frauscher, T. Frede and A. G. Zeimet, "Breast Cancer in Women: Evaluation of Benign and Malignant Axillary Lymph Nodes with Contrast-Enhanced Ultrasound," Ultraschall in der Medizin, Vol. 31, No. 1, 2010, pp. 63-67. doi:10.1055/s-0028-1109847

[20] I. J. Robertson, F. Hand and M. R. Kell, "FDG-PET/CT in the Staging of Local/Regional Metastases in Breast Cancer," Breast, Vol. 20, No. 6, 2011, pp. 491-494. doi:10.1016/j.breast.2011.07.002

[21] S. E. Harnan, K. L. Cooper, Y. Meng, S. E. Ward, P. Fitzgerald, D. Papaioannou, C. Ingram, E. Lorenz, I. D. Wilkinson and L. Wyld, "Magnetic Resonance for Assessment of Axillary Lymph Node Status in Early Breast Cancer: A Systematic Review and Meta-Analysis," European Journal of Surgical Oncology, Vol. 37, No. 11, 2011, pp. 928-936. doi:10.1016/j.ejso.2011.07.007

[22] E. Botteri, V. Bagnardi, A. Goldhirsch, et al., "Axillary
Lymph Node Involvement in Women with Breast Cancer: Does It Depend on Age?" Clinical Breast Cancer, Vol. 10, No. 4, 2010, pp. 318-321. doi:10.3816/CBC.2010.n.042

[23] H. Kennecke, R. Yerushalmi, R. Woods, et al., "Metastatic Behavior of Breast Cancer Subtypes," Journal of Clinical Oncology, Vol. 28, No. 20, 2010, pp. 3271-3277. doi:10.1200/JCO.2009.25.9820

[24] K. D. Voduc, M. C. Cheang, S. Tyldesley, et al., "Breast Cancer Subtypes and the Risk of Local and Regional Relapse," Journal of Clinical Oncology, Vol. 28, No. 10, 2010, pp 1684-1691. doi:10.1200/JCO.2009.24.9284

[25] M. J. van de Vijver, Y. D. He, L. J. van't Veer, et al., “A Gene-Expression Signature as a Predictor of Survival in Breast Cancer," The New England Journal of Medicine, Vol. 347, No. 25, 2002, pp. 1999-2009. doi:10.1056/NEJMoa021967

[26] A. Goldhirsch, W. C. Wood, A. S. Coates, R. D. Gelber, B. Thurlimann and H. J. Senn, "Strategies for SubtypesDealing with the Diversity of Breast Cancer: Highlights of the St Gallen International Expert Consensus on the Primary Therapy of Early Breast Cancer 2011," Annals of Oncology, Vol. 22, No. 8, 2011, pp. 1736-1747. doi:10.1093/annonc/mdr304

[27] P. Karlsson, B. F. Cole, B. H. Chua, K. N. Price, J. Lindtner, J. P. Collins, A. Kovács, B. Thürlimann, D. Crivellari, M. Castiglione-Gertsch, J. F. Forbes, R. D. Gelber, A. Goldhirsch and G. Gruber, for the International Breast Cancer Study Group, "Patterns and Risk Factors for Locoregional Failures after Mastectomy for Breast Cancer: An International Breast Cancer Study Group Report," Annals of Oncology, Vol. 23, No. 11, 2012, pp. 28522858. doi:10.1093/annonc/mds118

[28] B. Fisher, J. H. Jeong, S. Anderson, et al., "Twenty-FiveYear Follow-Up of a Randomized Trial Comparing Radical Mastectomy, Total Mastectomy, and Total Mastectomy Followed by Irradiation," The New England Journal of Medicine, Vol. 347, 2002, pp. 567-575. doi:10.1056/NEJMoa020128

[29] S. Latosinsky, T. S. Berrang, C. S. Cutter, R. George, I. Olivotto, T. B. Julian, A. Hayashi, C. Baliski, R. L. Croshaw, K. M. Erb and J. Chen, "CAGS and ACS Evidence Based Reviews in Surgery 40. Axillary Dissection versus No Axillary Dissection in Women with Invasive Breast Cancer and Sentinel Node Metastasis," Canadian Journal of Surgery, Vol. 55, No. 1, 2012, pp. 66-69.

[30] T. J. Whelan, I. A. Olivotto, I. Ackerman, et al., "NCICCTG MA20: An Intergroup Trial of Regional Nodal Irradiation in Early Breast Cancer," Journal of Clinical Oncology, Vol. 29, No. 15, 2011, Article ID: LBA1003.

[31] M. E. Straver, P. Meijnen, G. van Tienhoven, et al., "Sentinel Node Identification Rate and Nodal Involvement in the EORTC 10981-22023 AMAROS Trial," Annals of Surgical Oncology, Vol. 17, No. 7, 2010, pp 1854-1861. doi:10.1245/s10434-010-0945-Z

[32] W. P. Weber, M. Barry, M. M. Stempel, M. J. Junqueira, A. A. Eaton, S. M. Patil, M. Morrow and H. S. Cody 3rd, "A 10-Year Trend Analysis of Sentinel Lymph Node Frozen Section and Completion Axillary Dissection for 
Breast Cancer: Are These Procedures Becoming Obsolete?" Annals of Surgical Oncology, Vol. 19, No. 1, 2012, pp. 225-232. doi:10.1245/s10434-011-1823-Z

[33] I. van den Hoven, G. P. Kuijt, A. C. Voogd and R. M. H. Roumen, "High Intersystem Variability for the Prediction of Additional Axillary Non-Sentinel Lymph Node Involvement in Individual Patients with Sentinel NodePositive Breast Cancer," Annals of Surgical Oncology, Vol 19, No. 6, 2012, pp. 1841-1849. doi:10.1245/s10434-011-2169-2

[34] T. S. Berrang, M. Lesperance, P. T. Truong, C. Walter, A.
H. Hayashi and I. A. Olivotto, "Which Prediction Models Best Identify Additional Axillary Disease after a Positive Sentinel Node Biopsy for Breast Cancer?" Breast Cancer Research and Treatment, Vol. 133, No. 2, 2012, pp 695702. doi:10.1007/s10549-012-1991-y

[35] A. E. Giuliano, M. Morrow, S. Duggal and T. B. Julian, "Should ACOSOG Z0011 Change Practice with Respect to Axillary Lymph Node Dissection for a Positive Sentinel Lymph Node Biopsy in Breast Cancer?" Clinical and Experimental Metastasis, Vol. 29, No. 7, 2012, pp. $687-$ 692. doi:10.1007/s10585-012-9515-Z 